\title{
Delta- and gamma-activity of resting state EEG as one of markers of risk of depressive disorders in migrants of subpolar and polar regions of Siberia
}

\author{
Natalya Milakhina \\ ICG SB RAS, Novosibirsk, Russia \\ tashamilka@mail.ru \\ Alexandra Karpova \\ North-Eastern Federal University, \\ Yakutsk, Russia \\ karpova74@list.ru
}

\author{
Sergey Tamozhnikov \\ State Scientific-Research Institute of \\ Physiology \& Basic Medicine, \\ Novosibirsk, Russia \\ stam@physiol.ru \\ Alexander Savostyanov \\ ICG SB RAS, Novosibirsk, Russia \\ a-sav@mail.ru
}

\author{
Ekaterina Proshina \\ State Scientific-Research Institute of \\ Physiology \& Basic Medicine, \\ Novosibirsk, Russia \\ proshinaea@physiol.ru \\ Elena Afanaseva \\ North-Eastern Federal University, \\ Yakutsk, Russia \\ eb.afanaseva@mail.ru
}

\begin{abstract}
It is well known, the conditional rest state activates the functional rest network, including the brain default system (DMN) and the central executive network (CEN). At this stage of research, it can be assumed that both the process of adaptation to the polar climate and new social conditions, and the gradual reduction of the risk of depression among migrants, are due to functional changes within these two brain systems, as well as their functional relationships among themselves. Hypothetically, the increased activity of CEN is associated with a higher level of stress associated with moving to new living conditions
\end{abstract}

Keywords - EEG, depression, DMN, CEN, migrants, delta-rhythm, gamma-rhythm.

\section{Motivation and Aim}

\section{A. Motivation}

According to the WHO, today depression is one of the most common diseases worldwide, affecting more than 264 million people. Modern neuroscience is focused on the search for markers of mental illness for early diagnosis and rational treatment tactics.

\section{B. Aim.}

The aim of the study was to investigate the relationship between the indicators of electroencephalography in functional rest and predisposition to depressive disorders in labor migrants adapting to new climatic and social conditions (Republic of Sakha, Yakutia)

\section{Methods}

The experiment involved 50 young migrants and 40 indigenous people who were not previously supervised in psychoneurological dispensers and were not receiving psychological and psychiatric care at the time of the study. All migrants have moved to long-term residence in Yakutia from the southern regions (mainly Central Asia and Africa). The migrants were examined twice: Immediately after the move and six months after a non-visiting stay in Yakutia. In addition, the participants of the experiment filled the psychological questionnaires of Achenbach and the Beck Depression Inventory for further evaluation of the predisposition to depression.
EEG was recorded at rest with 128 channels of NeoRec amplifier, Russia. The evaluation of the current source density, connectivity and topological features of the rest EEG networks was made in the sLORETA software package.

\section{Results}

When comparing the results of psychological tests between migrants and indigenous people, it was found that migrants have an increased predisposition to develop depression and anxiety disorder immediately after the move, while according to the results of testing of the indigenous population, this trend was not observed. However, in the process of adaptation to new climatic and social conditions, the risk of these diseases in most migrants is reduced, which was revealed by the results of the same psychological tests conducted after six months. The high risk of depressive and anxiety disorders was associated with increased spectral power and connectivity in the delta and gamma rhythms bands in the medial dark and medial prefrontal cortex. Adaptation to the polar climate led to a decrease in the delta and gamma of spectral power, which correlated with a decrease in predisposition to depression.

\section{Conclusion}

Adaptation to the circumpolar climatic conditions is accompanied by the functional changes in the resting-state brain networks that can be revealed by the EEG analyses. The first stage of adaptation is accompanied by an increased activity of the central executive network (CEN) that is connected with the increased risk of developing of anxiety disorder. In later stage of adaptation, these changes become less pronounced that correlates with a reduced risk of anxiety and depressive disorders.

Acknowledgment

Supported by the Russian Foundation of Basic Research grants No. 18-29-13027, No. 18-415-140021 and the Budget project No. 0324-2019-0040-C-01. 